\title{
Determinación del agua retenida por el agregado en relación a las características superficiales y granulométricas
}

\author{
PEDRO ENRIQUE GRINSZPAN \\ Profesor Titular de la E. Otto Krause e I.T.S. \\ Integrante del L.E.M.E. y de la A.A.T.H. (*) \\ Quinta Reunión Técnica de la Asociación Argentina de Tecnología del Hormigón
}

LA PLATA - Buenos Aires/ARGENTINA

\begin{abstract}
$R E S U M E N$
El presente estudio comprende aspectos relacionados con la capacidad de los agregados para retener superficialmente parte del agua empleada en las mezclas de morteros $y$ hormigones.

Este relaciona dicha propiedad con las caracteristicas propias de los agregados. La capacidad de retención superficial resulta factor determinante en la dosificación de las mezclas de morteros y hormigones. Para ello se ha enjocado la relación entre granulometrias, módulo de finura y superficial envolvente, con relación al agua retenida superficialmente.

Se ha procurado desarrollar expresiones analiticas superficialmente generalizadas, para luego confrontarlas con resultados obtenidos en forma experimental en base a un procedimiento diseñado a tal efecto.

Posteriormente se relacionaron las variables entre si mediante expresiones que permitieron el desarrollo de funciones graficadas para correlacioner con los resultados experimentales, estableciéndose finalmente los limites de aceptación para este procedimiento.
\end{abstract}

\section{$S U M M A R Y$}

This study covers point relating to capacity of aggregates to superficially retain part of the water used in mortar and concrete mixtures.

It relates this property with the real characteristics of the aggregates. The superficial retention capacity is a decisive factor to determine dosage of mortar and concrete mixtures. The relation between granulometry, modulus of fineness and surrounding surface has accordingly been focused in relation to superficially retained water.

Sufficiently generalized analytic expressions have been developped, and later compared with results obtained in experiments based on a process designed for this purpose.

The variables are later inter-related using expressions that permitted graphic functions to be developped for correlation with the experimental results, and finally establishing acceptance limits for this process.

\section{INTRODUCCION}

Este trabajo pretende estudiar aspectos relacionados con la capacidad de los agregados para retener superficialmente agua, este aspecto resulta importante para la correcta determinación del agua de amasado, factor determinante en la dosificación de las mezclas de mortero y hormigones de cemento portland. El tema mereció la atención de destacados investigadores, cabe mencionar

(*) Profesor de Construcciones de Hormigón Armado, Estática y Estabilidad de las Construcciones, Proyectos de Hormigón Armado, y Tecnologia del Hormigón y Práctica de Obra, de los cursos regulares y para técnicos graduados. Integrante del L.E.M.E. de la F.R.A./U.T.N. Profesor de los Cursos de la A.A.T.H. 
expresamente a FERET, BOLOMEY y ABRAMS, quienes tempranamente relacionaron la retención superficial del agua por el agregado en función de su granulometría.

Se ha enfocado por un lado la relación entre granulometría, módulo de finura y superficie periférica, y por otra parte la relación entre ésta última y el agua "adsorbida" o retenida superficialmente.

Para la primera se procuró desarrollar una expresión analitica superficialmente generalizada, en tanto que para la segunda se abordó la vía de la determinación experimental, debiéndose diseñar para ello un procedimiento adecuado.

Posteriormente se formularon expresiones analiticas que permitieran vincular las variables entre si, partiendo para ello de la tabulación de los resultados y su representación gráfica. Por último se verificó si el grado de correlación obtenido puede ser considerado suficiente dentro de los límites impuestos al problema.

\section{CONSIDERACIONES INICIALES}

A efectos de simplificar el estudio, se consideró conveniente aceptar las siguientes premisas:

a) Partículas redondas idealmente esféricas.

b) Las partículas son lisas.

c) El agregado no es absorbente.

d) El agregado pasa totalmente el tamiz de $3 / 8$ ".

e) El agregado es preferentemente de granulometría contínua.

Con relación a los dos primeros puntos, se deberá introducir en cada caso los respectivos factores de corrección, por forma y por rugoridad:

\section{Factor de forma:}

$\mathrm{F}_{\mathrm{f}}=$ Vol. partícula $/ 0,785 \cdot D^{2}$.

$D=$ Diámetro de la esfera envolvente.

\section{Factor de rugoridad:}

$F_{\mathrm{r}}=$ Función de la textura superficial.

Respecto al tercero, se han utilizado agregados cuya observación es nula o insignificante.

En cuanto a los dos últimos, cabe agregar que siendo de gran preponderancia la incidencia del agregado fino en el cómputo de la superficie periférica o envolvente, en general aportan entre el 85 y el 90 por ciento del total, se ha optado por trabajar con arenas exclusivamente (es decir las fracciones pasantes del tamiz N. ${ }^{\circ} 4$ ) admitiéndose solamente una pequeña fracción comprendida entre los tamices \# 4 y de $3 / 8^{\prime \prime}$ a efectos de obtener alguna granulometría más elevada que amplie el espectro considerado. Se incluyeron algunas arenas de granulometría discontínua y muy limitada a efectos de observar si su comportamiento era similar al de las restantes.

Como es sabido, para una partícula esférica se tiene una superficie especifica $\left(S_{\mathrm{c}}\right)$ que depende del diámetro $(d)$ y del peso específico $\left(P_{\mathrm{e}}\right)$ del agregado, siendo entonces

$$
S_{\mathrm{c}}=6 /\left(P_{\mathrm{c}} \cdot d\right)
$$

se define a la superficie unitaria (por unidad de volumen absoluto) a la expresión

$$
S_{\mathrm{u}}=P_{\mathrm{c}} \cdot S_{\mathrm{c}}=6 / d
$$


resultando entonces la superficie envolvente o periférica $\left(S_{\mathrm{p}}\right)$ igual a

$$
S_{\mathrm{p}}=S_{\mathrm{u}} \cdot V_{\mathrm{r}} \text { (vol. absol. de las partículas) }
$$

Al trabajar con la superficie unitaria $\left(S_{\mathrm{u}}\right)$ se eliminan los problemas derivados de la variabilidad del peso específico.

Para describir la granulometría de una composición se utilizará el módulo de finura $(m)$ de Abrams y el tamaño límite superior de la serie de tamices A.S.T.M., correspondiente al menor tamiz que permite el pasaje del cien por ciento de la muestra $\left(d_{n}\right)$, éste último referido al número de orden $(n)$ de los tamices de dicha serie, en orden creciente, a partir del cero correspondiente al tamiz \# $200(74 \mu)$; éste número coincide con el módulo de finura de la fracción retenida sobre el tamiz considerándola como un cien por ciento. El tamaño limite superior (TLS) es siempre igual o superior al tamaño máximo nominal (TMN) correspondiente al menor tamiz de la serie que permite el pasaje de una cantidad no menor que el noventa y cinco por ciento.

Además se admitirá la sustitución de un perfil granulométrico real por otro ideal equivalente, de igual módulo $(m)$ de finura y constituido por una parábola de grado ere $(r)$, expresable en función del pasante $P_{\mathrm{i}}$ para cada tamaño $d_{\mathrm{i}}$ del siguiente modo

$$
\left(\% P_{\mathrm{i}} / 100\right)=\left(d_{\mathrm{i}} / d_{\mathrm{n}}\right)^{\mathrm{r}}=(2 \mathrm{r})^{\mathrm{r}-1}=a^{(\mathrm{i} \mathrm{-} \mathrm{n})}
$$

se cumplirá entonces que

$$
m=n-\frac{1-1 / a^{\mathrm{n}}}{1-1 / a}=n-\frac{1-A^{\mathrm{n}}}{1-A}
$$

siendo $A=1 / a=1 / b^{r}=(0,5)^{r}$

Por consiguiente, definido " $n$ " ( $p / e l$ TIS) y el módulo " $m$ " se determina iterativamente el valor de " $A$ " y a partir de éste el de " $r$ " siendo

$$
A=1-\frac{1-A^{\mathrm{n}}}{n-m}=(0,5)^{\mathrm{r}}
$$

luego:

$$
r=\operatorname{Lg} A /(-0,301)
$$

debiendo " $r$ " clasificarse entre los valores limites establecidos $\left(r \leqslant r_{\text {Lim }}\right.$ ?) o igualmente, el coeficiente de aptitud $(C A)$ dentro de los márgenes recomendables o aceptables, siendo $C A=6 \cdot(r-0,5)$ cuanto más próximo a cero, mejor (parábola cuadrática de Gessner).

$$
\left.\begin{array}{l}
(-1 \leqslant C A \leqslant+1): \text { RECOMENDABLE } \\
(-1,5 \leqslant C A \leqslant-1): \text { ACEPTABLE }
\end{array}\right\} \text { Según los dominios normalizados. }
$$

El empleo de las lineas ideales sustituyentes de los perfiles reales, permite intentar desarrollar expresiones analiticas para establecer la superficie unitaria de la composición ideal y de su equivalente real de igual módulo de finura y área de Hummel.

\section{ANTECEDENTES}

Tanto BOLOMEY como ABRAMS proponen expresiones útiles para la determinación del agua 
de amasado en función del agua requerida por el cemento en peso $\left(C_{\mathrm{p}}\right)$ y la retenida por el agregado en peso $\left(I_{\mathrm{p}}\right)$ del siguiente tipo:

$$
A_{\mathrm{a}}=A_{\mathrm{c}}+A_{\mathrm{r}}
$$

con exclusión del agua de absorción, ambos investigadores concuerdan que el primer término es del orden de $A_{\mathrm{c}}=$ (aprox.) $0,23 \cdot C_{\mathrm{p}}$ en cambio para el segundo término, correspondiente al agua de adsorción o retenida superficialmente, proponen:

ABRAMS:

$$
A_{\mathrm{r}}=\frac{2 \cdot k}{100} \cdot(8-m) \cdot I_{\mathrm{p}} \quad \operatorname{con} k=1 \text { a } 1,2
$$

BOLOMEY:

$$
A_{\mathrm{r}}=(0,8 \text { a } 1,2) \cdot N \cdot I_{\mathrm{p}} / d_{(\mathrm{mm})}^{2 / 3} \quad \operatorname{con} N=0,1
$$

convirtiéndose la expresión en una sumatoria cuando intervienen varios tamaños $(d)$ de agregados, en la práctica para cada fracción $d_{\mathrm{i}}$ retenida, el témino divisor pasa a ser

$$
d_{(\mathrm{mm})}^{2 / 3}=\left[d_{\mathrm{i}(\mathrm{mm})} \cdot d_{(\mathrm{mm}) \mathrm{i}+1}\right]^{1 / 3}
$$

puede transformarse en

$$
A_{\mathrm{r}}=(\text { aprox. }) \frac{0,10 \cdot S_{\mathrm{u}}^{2 / 3}}{6^{2 / 3}} \cdot I_{\mathrm{p}} \quad \text { con } S_{\mathrm{u}} \text { en } \mathrm{mm}^{2} / \mathrm{mm}^{3}
$$

Pueden igualarse ambas expresiones y obtener la siguiente fórmula de aplicación aproximada, pero fácil:

$$
S_{\mathrm{u}}=|2 / 3 \cdot(8-m) \cdot k|^{3 / 2} \text { en } \mathrm{mm}^{2} / \mathrm{mm}^{3}
$$

equivalente a

$$
S_{\mathrm{u}}=10 \cdot\left(S_{\mathrm{u}} \text { en } \mathrm{mm}^{2} / \mathrm{mm}^{3}\right) \text { en } \mathrm{cm}^{2} / \mathrm{cm}^{3}
$$

Sin embargo, ésta expresión constituye una aproximación limitada por su origen indirecto y para granulometrías elevadas debe descomponerse en sumatoria ponderada de las distintas fracciones intervinientes, dado que pierde sensibilidad para valores de " $n$ " cercanos a ocho y se anula al llegar a éste límite, por encima del mismo carece de sentido al dar valores negativos, por consiguiente:

$$
\left.S_{\mathrm{u}}=\mid \mathrm{SUM} r_{\mathrm{i}} \cdot S_{\mathrm{u}(\mathrm{i})}\right]_{0}^{\mathrm{n}}
$$

con $r_{\mathrm{i}}=$ retenido parcial correspondiente a una fracción.

Siendo en este caso más sencillo y exacto establecer:

$$
\left.S_{\mathrm{u}}=6 \cdot \mid \operatorname{SUM}\left(r_{\mathrm{i}} / d_{\mathrm{i}}\right)\right]_{0}^{\mathrm{n}}
$$

debiendo considerarse el tamaño medio de cada fracción $d_{\mathrm{i} \text { (medio) }}=\left(d_{\mathrm{i}} \cdot d_{\mathrm{i}+1}\right)^{1 / 2}$ dado que $d_{\mathrm{i}}$ es solamente un tamaño nominal de todas las partículas depositadas sobre dicho tamiz. 
Por otra parte puede recurrirse al concepto de tamaño único equivalente (TUE) de idéntico módulo $(m)$ de finura y por consiguiente responde a una fracción única $\left(d_{\mathrm{eq}}\right)$ con un escalón total del cien por ciento e igual área de Hummel, resulta entonces:

$$
\operatorname{Lg} d_{\text {eq }}(\mu)=1,872+0,301 \cdot m=(\#) \ldots d_{\text {eq }}(\mu)=10(\#)
$$

luego

$$
S_{\mathrm{u}}\left(d_{\text {eq }}\right)=(1,5 \text { a } 2,5) \cdot 6 / d_{\text {eq }}
$$

dependiendo de la extensión del perfil granulométrico y de su continuidad. Esta expresión resulta por lo tanto muy poco precisa para su empleo.

\section{CONSIDERACIONES TEORICAS}

De lo anterior se desprende lo dificultoso de obtener una expresión de la superficie unitaria $\left(S_{u}\right)$ en función del módulo de finura de Abrams si se prescinde del perfil granulométrico, resultando evidente que éste último debicra, cuanto menos, acotarse en caracteristica y extensión:

PERFIL GRANULOMETRICO:

$\left\{\begin{array}{l}\text { Característica }\left\{\begin{array}{l}\text { Continua } \\ \text { Discontinua }\end{array}\right. \\ \text { Extensión }\left\{\begin{array}{l}\text { Limites } \\ \text { Superior, TIS, “ } n " \\ \text { Inferior, "o" ó "j" }\end{array}\right.\end{array}\right.$

En principio nos referiremos a las graduaciones granulométricas continuas con $d_{\mathrm{i}}$ variando entre $\mathrm{i}=0$ a $n$, para las cuales resulta aceptable considerar perfiles de sustitución mediante curvas ideales, tomando para estas parábolas de grado " $r$ " (tal como se expone en las consideraciones iniciales 2.) y consecuentemente desarrollar expresiones analiticas válidas para dichas lineas y sus análogas. Para ello consideramos:

el pasante acumulado

$$
\% P_{\mathrm{i}} / 100=\left(b^{r}\right)^{\mathrm{i} \cdot \mathrm{n}}=a^{(\mathrm{i}-\mathrm{n})}=A^{-(\mathrm{i}-\mathrm{n})}
$$

el pasante parcial $p_{\mathrm{i}}=P_{\mathrm{i}}-P_{\mathrm{i} .1 .1}$ siendo entonces

$$
\% p_{\mathrm{i}} / 100=a^{(\mathrm{i}-1-\mathrm{n})}=\left[(a-1) / a^{1+\mathrm{n}}\right] \cdot a^{\mathrm{i}}
$$

siendo la suma de pasantes

$$
\operatorname{SUM} p_{\mathrm{g}, \mathrm{n}}(\%) / 100=1-a^{(\mathrm{g}-1-\mathrm{n})}
$$

que para $g=1$ resulta

$$
\operatorname{SUM} p_{(\mathrm{g}, \mathrm{n})}=1-P_{0}=1-a^{(\mathrm{n})}
$$

donde $P_{0}=a^{(-\mathrm{n})}$ es la ordenada de la parábola que corresponde al pasante acumulado del Tamiz \# $200(74 \mu)$, caracteristica de una curva ideal que pasa por el origen real de coordenadas. Esta dificultad fue oportunamente subsanada en la propuesta de ROTHFUCHS que traslada el origen al punto $d_{0}$. 
La superficie unitaria correspondiente al tamaño $d_{\mathrm{i},}$ es:

$$
S_{\mathrm{u}(\mathrm{i}-1)}=6 / d_{\mathrm{i}-1}=6 / d_{0} \cdot b^{\mathrm{i}-1}=\left(6 \cdot b / d_{0}\right) \cdot b^{-1}
$$

el producto por la cantidad retenida en el respectivo tamiz es

$$
\begin{aligned}
S_{\mathrm{u}(\mathrm{i}-1)} \cdot \% p_{\mathrm{i}} / 100= & \left(12 / d_{0}\right) \cdot b^{-1} \cdot \frac{a-1}{a^{1+\mathrm{n}}} \cdot a^{\mathrm{i}}=\frac{12}{d_{0}} \cdot \frac{a-1}{a^{1+\mathrm{n}}} \cdot a^{(\mathrm{i} / \mathrm{r})} \cdot a^{\mathrm{i}}= \\
= & \frac{12}{d_{0}} \cdot \frac{a-1}{a^{1+\mathrm{n}}} \cdot a^{(1-1 / \mathrm{r}) \mathrm{i}}=S_{(\mathrm{i}-1)}^{\prime}
\end{aligned}
$$

simplificando

$$
a^{(1-1 / \mathrm{r})}=a^{1} \cdot a^{1 / r}=a \cdot b^{-1}=a / 2
$$

y efectuando la sumatoria para todos los tamaños, tendremos:

$$
\begin{aligned}
\operatorname{SUM}_{(\mathrm{g}, \mathrm{n})} S_{(\mathrm{i}-1)}^{,} & =\frac{12}{d_{0}} \cdot \frac{a-1}{a^{1+\mathrm{n}}} \cdot \frac{(a / 2)^{\mathrm{n}+1}-(a / 2)^{\mathrm{g}}}{(a / 2)-1}= \\
& =1.611 \mathrm{~cm}^{2} \cdot \frac{a-1}{a^{1+\mathrm{n}}} \cdot \frac{(a / 2)^{\mathrm{n}+1}-(a / 2)^{\mathrm{g}}}{(a / 2)-1}
\end{aligned}
$$

La superficie unitaria total resultará del cociente por la sumatoria de los respectivos retenidos (o pasantes):

$$
\text { TOT. } S_{\mathrm{u}(\mathrm{g}, \mathrm{n})}=\frac{\operatorname{SUM}_{(\mathrm{g}, \mathrm{n})} S_{(\mathrm{g}, \mathrm{n})}^{\prime}}{\operatorname{SUM}_{(\mathrm{g}, \mathrm{n})} p_{\mathrm{i}}}=1.611 \frac{a-1}{(a / 2)-1} \cdot \frac{(a / 2)^{\mathrm{n}+1}-(a / 2)^{\mathrm{g}}}{a^{\mathrm{n}+1}-a^{\mathrm{g}}} \mathrm{cm}^{2} / \mathrm{cm}^{3}
$$

Si consideramos el tamaño medio de las particulas sobre cada tamiz, habrá que introducir un factor de corrección igual a $(2)^{-1 / 2}$ quedando finalmente:

$$
S_{\mathrm{u}(\mathrm{g}, \mathrm{n})}=1.139 \frac{a-1}{a^{\mathrm{n}+1}-a^{\mathrm{g}}} \cdot \frac{(a / 2)^{\mathrm{n}+1}-(a / 2)^{\mathrm{g}}}{(a / 2)-1} \cdot \mathrm{cm}^{2} / \mathrm{cm}^{2}
$$

Aplicando la expresión anterior a distintas situaciones, se puede obtener la siguiente tabla de valores:

\begin{tabular}{|c|c|c|c|c|c|c|}
\hline \multicolumn{7}{|c|}{ AGREGADOS CON (TLS) “ $n$ ” } \\
\hline \multirow{2}{*}{ GRADO “r” } & \multicolumn{2}{|c|}{$\mathrm{n}=4$} & \multicolumn{2}{c|}{$\mathrm{n}=6$} & \multicolumn{2}{c|}{$\mathrm{n}=9$} \\
\hline & $\mathrm{g}=0$ & $\mathrm{~g}=2$ & $\mathrm{n}=0$ & $\mathrm{n}=2$ & $\mathrm{n}=0$ & $\mathrm{n}=2$ \\
\cline { 2 - 7 } 0,2 & 373,03 & 156,34 & 237,76 & 93,26 & 132,12 & 48,39 \\
0,3 & 341,45 & 151,58 & 201,37 & 85,36 & 97,07 & 39,20 \\
0,5 & 284,75 & 142,37 & 142,37 & 71,19 & 50,34 & 25,17 \\
0,7 & 237,47 & 133,73 & 100,67 & 59,37 & 26,10 & 16,16 \\
\hline
\end{tabular}


Para una curva granulométrica $(j, n$ ") desplazada en forma paralela a otra línea $(g, n)$ tal que la diferencia $g-j$ resulta igual a la diferencia $n-n$ " entonces se puede establecer la siguiente relación entre ambas curvas, en función de la necesaria corrección por corrimiento en abscisas:

$$
S_{\mathrm{u}(\mathrm{i}-1)}=\frac{6 \cdot b}{1,41 \cdot d_{0}} \cdot b^{-\mathrm{i}}=1.139 \cdot 2^{-\mathrm{i}} \mathrm{cm}^{2} / \mathrm{cm}^{3}
$$

por consiguiente, tendremos:

$$
S_{\mathrm{u}(\mathrm{j}, \mathrm{n})}=S_{\mathrm{u}(\mathrm{g}, \mathrm{n})} \cdot 2^{\left(\mathrm{n}^{\mathrm{n}}-\mathrm{n}\right)}=S_{\mathrm{u}(\mathrm{g}, \mathrm{n})} \cdot 2^{(\mathrm{i}-\mathrm{g})}
$$

como consecuencia de que por cada corrimiento equivalente a una posición en la serie de tamices normalizados, los tamaños se duplican una vez y la superficie se modifica en relación inversa $\left({ }^{*}\right)$.

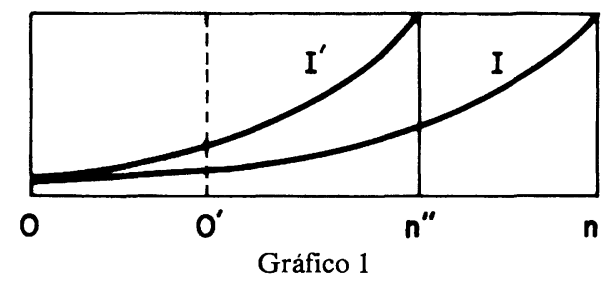

Considerando lo expuesto hasta ahora, veamos su aplicación a un ejemplo numérico, en el cual analizaremos la determinación de la superficie unitaria $\left(S_{\mathrm{u}}\right)$ en un agregado compuesto (III) con un perfil extendido entre los tamices $0-9$ y los componentes (I) y (II) extendidos entre los tamices $0-2$ y $2-9$, éste último como perfil desplazado paralelamente respecto a otro (II') situado entre 0-7. Consideramos que las distintas líneas son de grado $r=0,500$ (parábola cuadrática) con $a=2^{0,5}=1,4142$ y $A=1 / a=0,707$; se determina entonces:

III: $\quad S_{\mathrm{u}(0,9)}=1.139 \cdot \frac{1,4142-1}{\frac{1,4142}{2}-1} \cdot \frac{0,707^{10}-0,707^{0}}{1,4142^{10}-1,4142^{0}}=50,35 \mathrm{~cm}^{2} / \mathrm{cm}^{3}$

I:

$$
S_{\mathrm{u}(0,2)}=-1.611 \cdot \frac{0,707^{3}-1}{1,4142^{3}-1}=570 \mathrm{~cm}^{2} / \mathrm{cm}^{3}
$$

II':

$$
S_{\mathrm{u}(0,7)}=-1.611 \cdot \frac{0,707^{8}-1}{1,4142^{8}-1}=100,7 \mathrm{~cm}^{2} / \mathrm{cm}^{3}
$$

II:

$$
S_{\mathrm{u}(2 / 2,9)}=S_{\mathrm{u}(0,7)} \cdot 2^{-2}=100,7 \cdot 0,25=25,2 \mathrm{~cm}^{2} / \mathrm{cm}^{3}
$$

siendo:

$$
S_{\mathrm{u}(2.9)}=-1.611 \cdot \frac{0,707^{10}-0,707^{2}}{1,4142^{10}-1,4142^{2}}=25,17 \mathrm{~cm}^{2} / \mathrm{cm}^{3}
$$

${ }^{*}$ ) V. Interpretación y medición comparada de lineas granulométricas continuas. P. E. Grinszpan/4a. R.Tca., A.A.T.H.Mendoza 1079. 
por otra parte, sobre la linea compuesta (III) tendremos los siguientes pasantes y retenidos:

$$
\begin{array}{lr}
\% p_{2} / 100=1,4142^{2} \cdot(1,4142-1) / 1,4142^{10}=0,0259 & \\
\% p_{1} / 100=1,4142^{1} \cdot(1,4142-1) / 1,4142^{10}=0,01883 & +=0,0442 \\
\% P_{2} / 100=1,4142^{(2-9)}=0,0884 & \underline{0,9116} \\
\% R_{2} / 100=1-0,0884=0,9116 & 0,9558 \% \text { TOT } / 100
\end{array}
$$

Efectuando la mezcla de los valores de $S_{\mathrm{u}(0,2)}$ y $S_{\mathrm{u}(2 / 2,9)}$ en función de las respectivas proporciones, se tiene:

$$
\begin{gathered}
S_{\mathrm{u}(0,9)}=\left(0,0442 S_{\mathrm{u}(0,2)}+0,9116 S_{\mathrm{u}(2 / 2,9)}\right) / 0,9558=(0,0442 \cdot 570+0,9116 \cdot 25,2) / 0,9558= \\
=48,166 / 0,9558=50,39=\text { aprox. } 50,35 \mathrm{~cm}^{2} / \mathrm{cm}^{3}
\end{gathered}
$$

el error resulta del orden de $0,8 \%$ despreciable a todos los efectos prácticos y consecuencia de redondeos del cálculo numérico.

Gráfico 2.-Ubicación de las lineas consideradas en el ejemplo: I, II, II' y III; con " $n=9$ ".

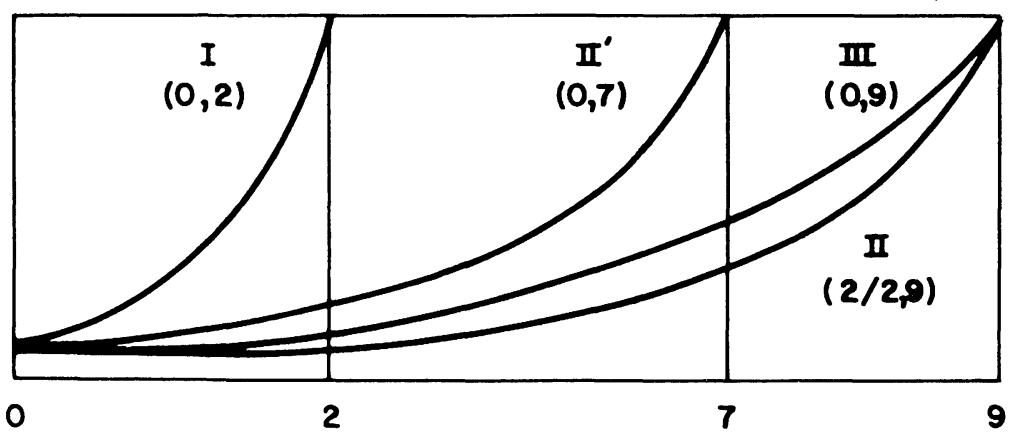

\section{AJUSTES}

Debe tenerse en consideración que al adoptar como líneas ideales a familias de parábolas (de distinto grado " $r$ ") que pasan por el origen real de coordenadas y no por el vértice inferior izquierdo de la gráfica, en correspondencia con la posición del Tamiz \# 200 (74 $\mu$ ) o número de orden cero, el retenido total para dicha abcisa es menor del cien por ciento, es decir $R_{0}<100 \%$ y consecuentemente $P_{0}>0 \%$, pero dado que para los agregados tendremos siempre $d>d_{0}$, resulta necesario introducir una corrección en las expresiones anteriores. Este problema quedaría obviado si se adoptase las lineas ideales propuestas por ROTHFUCHS, consistentes en parábolas desplazadas hasta el mencionado vértice, lograndose asi $R_{0}=100 \%$ $\left(P_{0} \%\right)$, pero la expresión que formula el perfil de esas líneas complica inconvenientemente los desarrollos analíticos.

Quedan pues, dos alternativas que permiten corregir la situación observada, mediante la primera se modifican todas las ordenadas mientras que, con la segunda, se ajusta únicamente la última corrrespondiente al retenido sobre el último tamiz, es decir el \#200, cambiando $r_{0}$ hasta el límite en que $R_{0}$ resulta igual al cien por ciento.

Veremos a continuación ambas alternativas y las consecuencias emergentes de éstas. 


\subsection{Corrección total de ordenadas}

El factor de corrección resulta igual a la relación entre la ordenada total y la original para la posición cero $(0)$, siendo:

$$
F C=100 / \% R_{0}=1 /\left(1-A^{\mathrm{n}}\right)=a^{\mathrm{n}} /\left(a^{\mathrm{n}}-1\right)=a^{\mathrm{n}+1} /\left(a^{\mathrm{n}+1}-a\right)
$$

Resultando

$$
S_{\mathrm{u}(0 / 1, \mathrm{n})}^{\text {corr. }}=F C \cdot S_{\mathrm{u}(0 / 1, \mathrm{n})}
$$

finalmente

$$
S_{\mathrm{u}(0 / 1, \mathrm{n})}^{\text {corr. }}=1.139 \cdot \frac{a-1}{a / 2-1} \cdot \frac{(a / 2)^{\mathrm{n}+1}-(a / 2)}{\left(a^{\mathrm{n}+1}+a\right)^{2}} \cdot a^{\mathrm{n}+1}
$$

\subsection{Correccion de la ordenada final}

Retomando la expresión sumatoria de las superficies correspondientes a las distintas fracciones (v. pg. 7) que da

$$
\operatorname{SUM}_{(\mathrm{g}, \mathrm{n})} S_{(\mathrm{i}-1)}^{\prime}=1.139 \cdot \frac{a-1}{a^{1+\mathrm{n}}} \cdot \frac{(a / 2)^{\mathrm{n}+1}-(a / 2)^{\mathrm{g}}}{(a / 2)-1}
$$

tomando los límites $(0 / 1, n)$ se tiene

$$
1.139 \cdot \frac{a-1}{a^{1+\mathrm{n}}} \cdot \frac{(a / 2)^{\mathrm{n}+1}-(a / 2)^{\mathrm{g}}}{(a / 2)-1}
$$

y que corresponde a la superficie del conjunto $R_{0}$ faltando cumputar la cantidad de material que en la curva teórica (parábola de grado " $r$ ") pasa el Tamiz \# 200 (tamiz 0), consistiendo la corrección, justamente, en incluirlo en el cómputo, por consiguiente se tendrá un incremento igual a

$$
\text { DIF } S_{\mathrm{u}(0)}=\frac{6}{d_{0} \cdot(2)^{1 / 2}} \cdot \frac{2}{2} \cdot(a)^{-\mathrm{n}}=1.139 \cdot \frac{(a)^{-\mathrm{n}}}{2}
$$

que sumado a la expresión anterior y dividido por la suma de las fracciones, ahora igual a $\operatorname{SUM} p_{(0, \mathrm{n})}=1$ da finalmente la superficie unitaria corregida para el conjunto $(0, n)$ :

$$
S_{u(0 / 1, \mathrm{n})}^{\text {corr. }}=1.139 \cdot(1 / a)^{\mathrm{n}} \cdot\left(\frac{1}{2}-\frac{[1-(1 / a)] \cdot\left[1-(a / 2)^{\mathrm{n}}\right]}{1-(2 / a)}\right)
$$

$\operatorname{con} A=1 / a$

$$
S_{\mathrm{u}(0 / 1 . \mathrm{n})}^{\text {corr. }}=1.139 \cdot A^{\mathrm{n}} \cdot\left(\frac{1}{2}-\frac{(1-A) \cdot\left[1-1 /\left(2^{\mathrm{n}} \cdot A^{\mathrm{n}}\right)\right]}{1-2 \cdot A}\right)
$$




\subsection{Diferencias entre ambas alternativas}

En el primer caso (5.1.) el procedimiento consiste en que la línea tenga una rotación alrededor del vértice superior derecho de la gráfica, hasta que el extremo inferior alcance el vértice inferior izquierdo, con ello se produce una modificación en todos los valores parciales correspondiente al conjunto total, en cambio el segundo caso (5.2.) mantiene inalterada la línea por sobre $d_{1}$ girando únicamente la parte restante de la curva, tramo $d_{0}-d_{1}$ hasta completar la ordenada $R_{0}=1$, con ello se logra efectuar la corrección respetando en su mayor parte la hipótesis inicialmente establecida para la linea ideal. Por consiguiente consideramos más ajustada ésta última expresión y la adoptamos para las consideraciones subsiguientes.

$$
S_{\mathrm{u}(0 / 1, \mathrm{n})}=1.139 \cdot A^{\mathrm{n}} \cdot\left(\frac{1}{2}-\frac{(1-A) \cdot\left[1-1 /\left(2^{\mathrm{n}} \cdot A^{\mathrm{n}}\right)\right]}{1-2 \cdot A}\right) \mathrm{en}^{2} \dot{2} / \mathrm{cm}^{3}
$$

Los subindices que acompañan a la expresión $S_{\mathrm{u}}$ indican en primer término el nuevo origen adoptado (correspondiente a la posición " 0 " del Tamiz \# 200) y luego a los tamices extremos entre los que se considera el material pasante (pasan por el " $n$ " y sucesivamente los subsiguientes hasta el "1" inclusive).

La expresión así hallada establece valores que coinciden satisfactoriamente con los determinados analizando por separado cada fracción cnstituyente de una composición granulométrica, teniendo a su favor las ventajas derivadas de un cálculo único integrado mediante una sola expresión, la que es aplicable mediante el criterio de utilizar las líneas ideales equivalentes a los perfiles granulométricos reales.

\section{ESTUDIO EXPERIMENTAL}

Este trabajo se complementó mediante el estudio experimental de diversas muestras de arenas naturales, de composición original y compuestas, que cubren un espectro de granulometrías entre 1,04 (módulo de Abrams) y 4,84 como máximo, con un total de diez agregados distintos, siete de los cuales corresponden a perfiles de graduaciones continuas, las tres restantes son fráncamente discontinuas. Estas últimas se caracterizan por una deficiente estructura granular, siendo sus pesos unitarios en estado compactado $\left(P_{\mathrm{u}} c\right)$ sumamente bajos en relación con los agregados del primer grupo.

El estudio consistió en materializar un dispositivo de ensayo que permitiera apreciar la "retención superficial de agua" en base a un procedimiento que resultase los suficientemente sencillo como para admitir su traslado a las condiciones de obra, y efectuar suficientes determinaciones como para asegurar la estabilidad de los resultados.

En primer término se presentaron dos opciones para dichas determinaciones, a saber:

1) medir el tiempo $(t)$ en función de una retención de agua determinada; o

2) medir la retención de agua (AR) en función de un tiempo determinado

Se optó por esta segunda alternativa, por considerarla más práctica, decidiendose establecer una escala de tiempos que asegurase una "retención estabilizada" del agua.

Se estableció cantidades normalizadas de agregado y de agua pasante por éste, utilizándose el agregado en condiciones de S.S.S., las experiencias se efectuaron en laboratorio en ambiente húmedo. 


\subsection{Procedimiento adoptado}

Se adoptó un dispositivo constituido por un juego de tamices \# 100 y \# 50 con fondo y tapa, colocándose una muestra de arena de $2.400 \mathrm{~g}$ sobre el tamiz menor, una vez distribuida uniformemente se compacta mediante diez caidas o golpes del tamiz sobre la mesa de trabajo desde una altura de $10 \mathrm{~cm}$, si como consecuencia del procedimiento descrito, se constituyera una fracción residual pasante por el tamiz \# 100, la misma se colocará parejamente sobre la muestra, finalmente ésta deberá alisarse superficialmente. Se coloca encima el tamiz \#50 y ambos sobre el recipiente de fondo, a continuación se vierte $100 \mathrm{~g}$ de agua uniformemente sobre la superficie del tamiz \# 50 -que actúa como distribuidor del liquido- y se realizan las mediciones de retención de agua en una secuencia de tiempos fijos correspondientes a los 5', 10', 20', 30' y 45'. Como consecuencia de haber observado excesivas variaciones en las mediciones correspondientes a un mismo agregado y notándose que entre las dos primeras observaciones se originaban significativas precipitaciones independientes del efecto de adsorción, se incorporó al proceso, en correspondencia con la primera y con la última observación, un movimiento del tamiz destinado a asegurar en forma normalizada la decantación del líquido no retenido superficialmente y que quedaba en espacios vacíos de la estructura granular. Dicho movimiento consiste en efectuar una rotación de $360^{\circ}$ con el tamiz inclinado satisfactorio para anular la mencionada anomalia. Luego de efectuados numerosos ensayos quedó evidenciado que los valores de retención se estabilizaban ya a los 10' y que la pérdida posterior resultaba del orden del uno por ciento y por consiguiente no justificaba la extensión de los tiempos de ensayo, decidiéndose entonces acortar los mismos, limitando la última lectura a los 20', al cabo de los cuales se efectúa la segunda rotación (de $360^{\circ}$ en un plano de $45^{\circ}$ ) con el tamiz \# 100.

\subsection{Estudios efectuados}

Se estudiaron diez muestras distintas de arenas, ocho principales y dos complementarias, correspondientes a:

Ciento veinte ensayos, diez preliminares de ajuste, y los ciento diez restantes para las mediciones, obteniéndose medias y dispersiones standard que para nueve muestras dieron coeficientes de variación entre el tres y el seis por ciento, y para la restante (Mf: 4,84) el diez y seis por ciento.

\begin{tabular}{|c|c|c|c|c|c|c|c|c|c|}
\hline TIPO & $(\mathrm{mf})$ & "n" & A & $\mathbf{r}$ & $\mathbf{S}_{\mathrm{u}}$ & $\emptyset_{\mathrm{ep}}$ & $\mathbf{A R}$ & $S_{u}^{\text {eq }}$ & $A R /\left(S_{u}\right)^{2 / 3}$ \\
\hline I. 1 & 1,04 & 4 & 0,80 & 0,32 & 365 & $150 \mathrm{u}$ & 488,2 & 392 & 9,56 \\
\hline I. 2 & 2,55 & 4 & 0,32 & 1,64 & 118 & 440 & 475,3 & 138 & $19,8(+)$ \\
\hline I. 3 & 3,84 & 5 & 0,14 & 2,84 & 43 & 1.070 & 254,3 & 56 & $20,9(+)$ \\
\hline I. 4 & 1,76 & 4 & 0,62 & 0,69 & 238 & 250 & 430,2 & 238 & 11,2 \\
\hline 1.5 & 2,44 & 5 & 0,66 & 0,60 & 185 & 400 & 359,4 & 149 & 11,1 \\
\hline I. 6 & 3,22 & 5 & 0,45 & 1,15 & 91 & 690 & 384,2 & 87 & $19,0(+)$ \\
\hline 1.7 & 2,29 & 5 & 0,69 & 0,54 & 205 & 360 & 396,4 & 165 & 11,4 \\
\hline I. 8 & 4,84 & 7 & 0,54 & 0,89 & 44 & 2.130 & 155,6 & 28 & 12,5 \\
\hline I.' & 1,80 & & & & & 260 & 335,8 & 231 & 9 \\
\hline I." & 2,11 & & & & & 321 & 424,0 & 186 & 13 \\
\hline
\end{tabular}

Los agregados (+) identificados como I.2, I.3, e I.6 corresponden a estructuras granulométricas discontinuas, los agregados I.' e I." son muestras complementarias. Las muestras principales continuas cumplen muy bien la expresión de BOLOMEY que relaciona el agua retenida AR con la superficie envolvente potenciada a los dos tercios, se observa que los resultados de la última 
columna de la tabla precedente son casi constantes, aproximadamente 11,1 (entre 9,6 y 12,5). Debe tenerse presente que los valores del agua retenida AR son los correspondientes a los $2.400 \mathrm{~g}$ de agregado $\left(I_{\mathrm{p}}\right)$ de la muestra. La superficie unitaria $S_{\text {u }}$ se ha expresado en $\mathrm{cm}^{2} / \mathrm{cm}^{3}$.

Utilizando la expresión de $S_{\mathrm{u}}$ obtenida en la pág. 5 en base a las expresiones de Bolomey, se tiene para los agregados anteriores los siguientes valores:

$95,466,155,181,068,154,370,929,280,2$ y 74,3 en $\mathrm{cm}^{2} / \mathrm{cm}^{3}$ en el orden de la tabla anterior.

Relacionando los valores obtenidos experimentalmente de $\mathrm{AR}$ con el mf del agregado se obtiene la siguiente expresión:

$$
\operatorname{AR}(\mathrm{g})=(0,245-0,037 \cdot \mathrm{mf}) \cdot I_{\mathrm{p}}
$$

$\operatorname{con} I_{\mathrm{p}}=2.400 \mathrm{~g}$.

$$
I_{\mathrm{p}} \cdot \mathrm{AR}(\mathrm{g})=588-88,5 \cdot \mathrm{mf}
$$

Relacionando los valores teóricos y experimentales del AR se obtienen los siguientes valores

\begin{tabular}{|c|c|c|c|c|c|c|c|c|}
\hline TIPO & $(\mathrm{mf})$ & $\begin{array}{c}\mathbf{1}=\mathbf{A R} \\
\text { ens. }\end{array}$ & $\begin{array}{c}2=\mathrm{AR} \\
\text { Bolomey }\end{array}$ & $\begin{array}{l}3=A R \\
\text { Abrams }\end{array}$ & $\begin{array}{c}4=A R(\#) \\
\text { Ecuación }\end{array}$ & $2 / 1$ & $3 / 1$ & $4 / 1$ \\
\hline I. 1 & 1,04 & 0,20 & 1,10 & 0,14 & 0,207 & 5,50 & 0,70 & 1,03 \\
\hline I. 2 & 2,55 & 0,20 & 0,52 & 0.11 & 0,15 & 2,60 & 0,55 & 0,75 \\
\hline I. 3 & 3,84 & 0,11 & 0,26 & 0,08 & 0,10 & 2,36 & 0,73 & 0,91 \\
\hline I. 4 & 1,76 & 0,18 & 0,83 & 0,12 & 0,18 & 4,61 & 0,67 & 1.00 \\
\hline I. 5 & 2,44 & 0,15 & 0,70 & 0,11 & 0,155 & 4.67 & 0,73 & 1.03 \\
\hline I. 6 & 3,22 & 0,16 & 0,43 & 0,095 & 0,126 & 2,69 & 0,59 & 0,79 \\
\hline I. 7 & 2,29 & 0,165 & 0,75 & 0,11 & 0,16 & 4,54 & 0,67 & 0,97 \\
\hline I. 8 & 4,84 & 0,065 & 0,27 & 0,06 & 0,066 & 4,15 & 0,92 & 1,01 \\
\hline
\end{tabular}
para las ocho muestras principales:

Observese la excelente correlación de los valores obtenidos mediante la ecuación lineal (\#), de la pág. anterior, con los obtenidos experimentalmente, respecto a los determinados por Abrams pueden ser considerados aceptables, en cambio los hallados por Bolomey resultan con una mayor dispersión.

Efectuando cálculos de regresión lineal entre las distintas variables analizadas, AR (ens.), mf y $S_{\mathrm{u}}$ (ecuación), se obtienen las siguientes rectas de regresión y respectivos coeficientes de correlación $(\# R)$ :

$$
\begin{array}{lrll}
\mathrm{AR}= & 603,3-85,67 & \mathrm{mf} & \\
\mathrm{mf}= & 6,30-0,0097 \mathrm{AR} & \# R=-0,91 \\
\mathrm{AR}= & 248,2+0,74 & S_{\mathrm{u}} & \\
S_{\text {u }}=-98,95+0,71 & \mathrm{AR} & \# R=0,72 \\
S_{\mathrm{u}}=394,2-84,84 & \mathrm{mf} & \\
\mathrm{mf}= & 4,37-0,01 & S_{\mathrm{u}} & \# R=-0,924
\end{array}
$$


Dado que las muestras de agregados de módulos de finura mf igual a 2,55 y 3,22 corresponden a los agregados de granulometria discontinua más atípicos, se optó por efectuar una nueva regresión excluyendo los valores pertenecientes a estos agregados, obteniendo así las siguientes expresiones:

$$
\begin{array}{lrll}
\mathrm{AR}= & 583,2-87,28 & \mathrm{mf} & \\
\mathrm{mf}= & 6,67-0,011 & \mathrm{AR} & \# R=-0,9975 \\
\mathrm{AR}= & 176,3+0,94 & S_{\mathrm{u}} & \\
S_{\mathrm{u}}=-150,3+0,95 & \mathrm{AR} & \# R=+0,945 \\
S_{\mathrm{u}}=407,4-84,16 & \mathrm{mf} & \\
\mathrm{mf}= & 4,65-0,01085 & S_{\mathrm{u}} & \# R=-0,955
\end{array}
$$

Estas expresiones demuestran un excelente ajuste respecto a los valores establecidos para las distintas muestras.

\subsection{Fórmulas propuestas}

Finalmente se ha graficado las distintas funciones, trazando posteriormente lineas de ajuste que presentarán la mayor aproximación, estimando satisfactoria la regresión lineal para relacionar el agua retenida $\mathrm{AR}$ con el modulo de finura $\mathrm{mf}$, en cambio para relacionar el módulo de finura $\mathrm{mf}$ con la superficie unitaria $S_{\text {u }}$ y ésta con el agua retenida AR se ha encontrado más apropiado utilizar líneas curvas, definiéndose las siguientes funciones:

$$
\begin{gathered}
\operatorname{AR}\left(p / I_{\mathrm{p}}=2.400 \mathrm{~g}\right)=588-88,5 \mathrm{mf} \\
\mathrm{mf}=6 /\left(e^{\mathrm{U} / 209}\right) \\
\operatorname{AR}\left(p / I_{\mathrm{p}}=2.400 \mathrm{~g}\right)=588-531 /\left(e^{\mathrm{U} / 209}\right)
\end{gathered}
$$

siendo $U=S_{\mathrm{u}}$ en $\mathrm{cm}^{2} / \mathrm{cm}^{3}$

Finalmente de la segunda expresión se obtiene también:

$$
S_{\mathrm{u}}=U=375-480 L_{\mathrm{g}} \mathrm{mf}
$$

Todas estas funciones se han representado en las tres láminas siguientes, en las cuales se observa la dispersión que presentan los tres agregados de estructura granulométrica discontinua.

Gráfico 3.-Agua retenida (según ensayos) en relación al módulo de finura (Abrams) obtenido por tamizado.

Rectas de regresion c) todos los valores (tr. fuerte cortado).

a) Respecto a mf: 7,04 y 603,3.

b) Respecto a AR: 6,30 y 652,6.

Rectas de regresión sin valores atipicos (tr. fino guión punto).

c) Respecto a mf: 6,68 y 583.2.

d) Respecto a AR: 6,67 y 584,3.

Correlación: - 0,91 (t. val.) y 0,9975.

Recta de aproximación, ecuación:

$$
\mathrm{AR}=588-88,5 \cdot \mathrm{mf} \text {. }
$$

Puntos adoptados p/la recta: 1,04/488,2; 2,29/396,4; $4,84 / 155,6$.

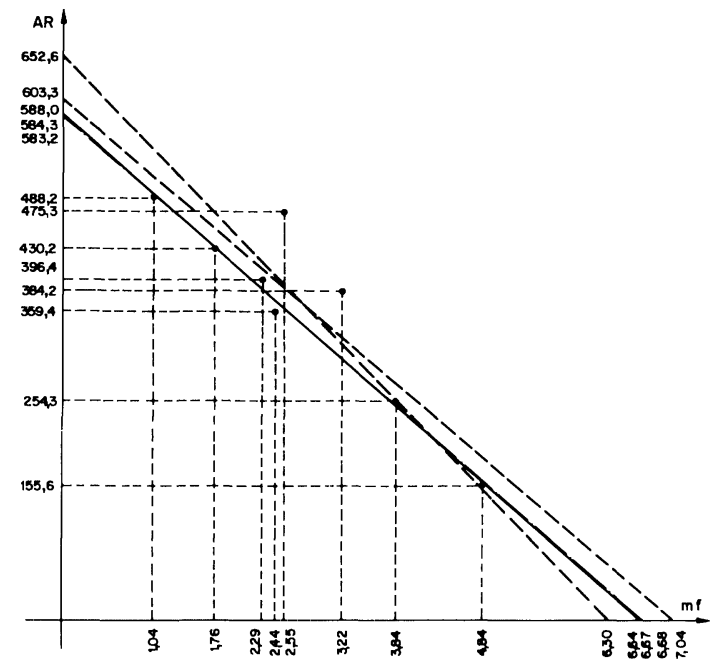




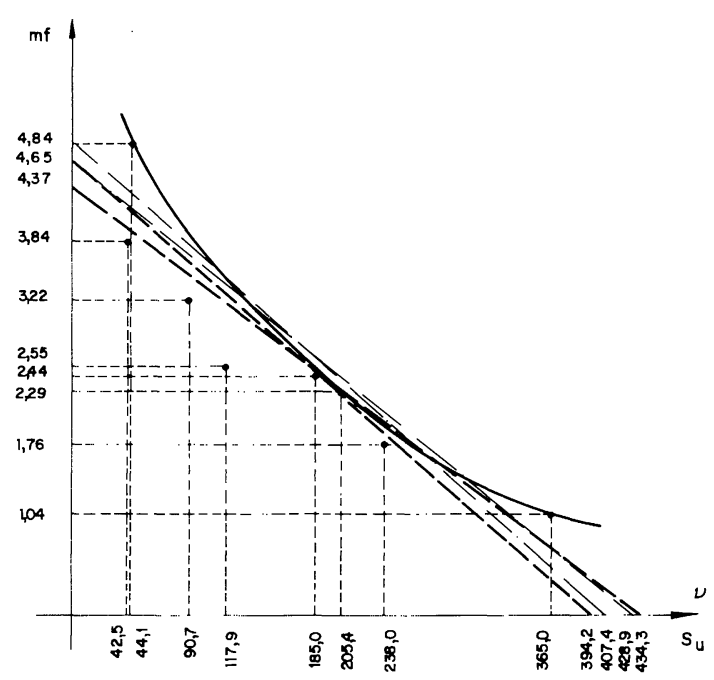

Gráfico 4.- Relación entre el módulo de finura (Abrams) y la superficie unitaria determinada en la forma analítica.

El gráfico representa:

1) Puntos aislados obtenidos $p /$ ensayos.

2) Rectas de regresión respecto a ambos ejes considerados todos los valores (trazos fuertes) y sin valores atipicos (trazos finos).

3) Curva de aproximación considerando dos puntos extremos y uno intermedio.

Ecuación:

$$
\mathrm{mf}=6 /\left(e^{\mathrm{U} / 209}\right)
$$

Correlación:

$$
\begin{aligned}
& -0,924 \text { (t. val.) y } \\
& -0,955 \text { (s/val. atípicos). } \\
& U=\text { Cte. } A^{\mathrm{n} 0} \cdot F(A, n)
\end{aligned}
$$

Recta c/todos los valores (trazo fuerte cortado):

a) Respecto a U : 434,3 y 4,37.

b) Recspecto a mf: 394,2 y 4,65 .

b) Respecto a mf: 394,2 y 4,65.

Recta sin los valores atípicos (tr. fino guión y punto).

c) Respecto a U : 428,9 y 4,65.

d) Respecto a mf: 407,4 y 4,84 .

Curva de aproximación, puntos adoptados: 44,1/4,84;

205,4/2,29; 365/1,04.

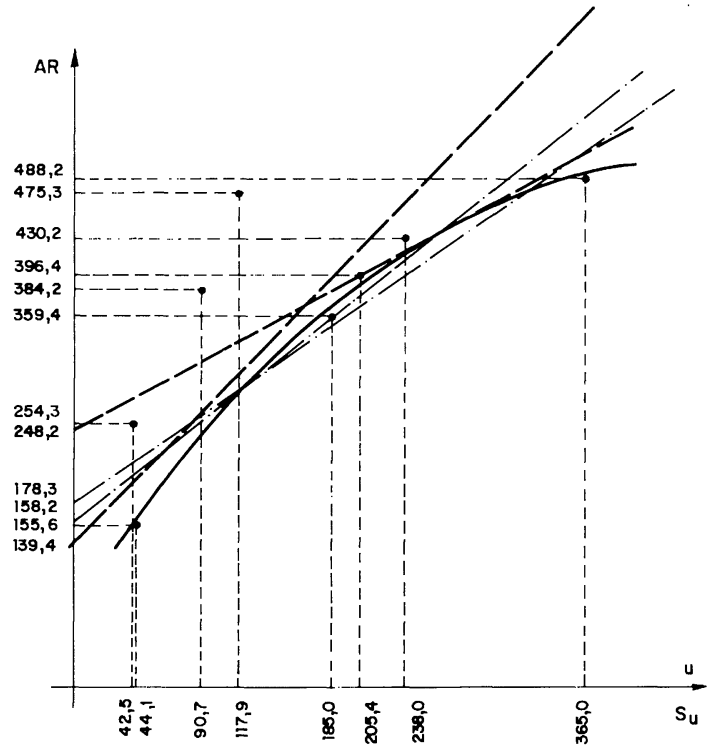

Gráfico 5.-Agua retenida (según ensayos) en relación a la superficic unitaria determinada en forma analitica.

AR: en g por $2.400 \mathrm{~g}$ agregado.

Rectas de regresión: c/todos los valores (tr. fuerte cortado).

a) Respecto a U, $\mathrm{AR}=248,2$.

b) Respecto a $\mathrm{AR}, \mathrm{AR}=138,4$.

Sin valores atipicos (tr. fino guión punto).

c) Respecto a U, $A R=178,3$.

d) Respecto a AR, AR $=158,2$.

Curva de aproximación, ecuación:

$$
\mathrm{AR}=588-531 /\left(e^{\mathrm{U} / 209}\right)
$$

Puntos adoptados p/la curva: 44,1/155,6; 205,4/396,4; $365,0 / 488,2$

Correlacion: 0,72 (t. val.) y 0,945 (s/val. atip.).

\section{CONSIDERACIONES FINALES}

El estudio realizado permite establecer relaciones satisfactorias entre las variables consideradas. Mediante el criterio de sustituir los perfiles granulométricos reales por líneas ideales equivalentes, de igual módulo de finura mf e igual tamaño límite superior TLS, definidas mediante parábola de grado " $r$ ", resulta posible aplicar sencillas expresiones de cálculo que vinculan la superficie unitaria $S_{\mathrm{u}}$ con el módulo de finura $\mathrm{mf}$ y el agua retenida AR superficialmente por dicho agregado.

Resulta de particular interés la significativa coincidencia que se verifica entre el agua retenida AR y la expresión $\left(S_{\mathrm{u}}\right)^{2 / 3}$ que deriva de la expresión $(1 / d)^{2 / 3}$ propuesta originalmente por Bolomey y que ha resultado de probada eficacia. Sin embargo se evidencia que las composiciones granulométricas discontinuas presentan un comportamiento anómalo con una retención aparentemente mucho más elevada, del orden de un ochenta por ciento mayor que las continuas. 
Para las tres composiciones $(+)$ de esta clase, se observa en la última columna de la primera tabla de valores comparativos:

Valor medio $\mathrm{AR} /\left(S_{\mathrm{u}}\right)^{2 / 3}=19,9$ contra 11,1 de las continuas, es decir $100(19,9-11,1)$ / $/ 11,1=80 \%$.

Esta diferencia hallaría su explicación en la constitución de una estructura granular deficientemente compacta, dando lugar por consiguiente a la cabida de una mayor cantidad de líquido, en tal sentido debe observarse la coincidencia con un peso unitario compactado menor que el determinado en las composiciones continuas.

El trabajo hasta aquí realizado, presenta resultados de interés práctico, considerándose que el tema no ha sido agotado y que una posterior ampliación del mismo permitirá profundizar las conclusiones y extenderse sobre la incidencia de la estructura granular y su compacidad, especialmente en lo relativo a los perfiles granulométricos discontinuos, que presentan un comportamiento nétamente diferenciado.

\section{A G R A D E CIMIEN T O}

El autor agradece muy especialmente al Ing. José F. Colina, quien estimuló la iniciación de este trabajo, al Ing. M. Wainsztein por las valiosas sugerencias brindadas para este tema y al Ing. Robewrto P. Echarte por posibilitar las tareas desarrolladas en el L.E.M.E.-F.R.A./U.T.N.

Agradece, asimismo, a los Sres. Jorge Muiño y Daniel Bascoy la laboriosa y dedicada colaboración en las tareas de laboratorio, al Sr. Daniel N. Pérez en el proceso y graficación numérica.

Por último, agradece al Laboratorio de Ensayos Industriales y personal del mismo, en la E. Otto Krause, por la tarea desarrollada durante los años 1980 y 1981, al laboratorio de Ensayo de Materiales y Estructuras (I.E.M.E.) y personal del mismo, de la Ftad. Regional Avellaneda de la Universidad Tecnológica Nacional, por la tarea efectuada durante el año 1982 